\title{
Nonmodal Linear Stability of the Schwarzschild Black Hole
}

\author{
Gustavo Dotti* \\ Facultad de Matemática, Astronomía y Física, Universidad Nacional de Córdoba, \\ Instituto de Física Enrique Gaviola, Conicet. Ciudad Universitaria, 5000 Córdoba, Argentina \\ (Received 20 October 2013; published 14 May 2014)
}

\begin{abstract}
A proof is given that the space $\mathcal{L}$ of solutions of the linearized vacuum Einstein equation around a Schwarzschild black hole is parametrized by two scalar fields, which are gauge invariant combinations of perturbed algebraic and differential invariants of the Weyl tensor and encode the information on the odd (-) and even $(+)$ sectors $\mathcal{L}_{ \pm}$. These fields measure the distortion of the geometry caused by a generic perturbation and are shown to be pointwise bounded on the outer region $r \geq 2 M$.
\end{abstract}

DOI: 10.1103/PhysRevLett.112.191101

PACS numbers: 97.60.Lf, 04.20.-q

Introduction.-The formation of black holes in gravitational collapse is a fascinating prediction of Einstein's general relativity, which is backed by growing astrophysical evidence of their existence and abundance in the Universe. The mathematical modeling of these objects, however, is a field where a number of crucial problems are still open, the most salient ones being ruling out the alternative of formation of a naked singularity in gravitational collapse, that is, establishing the validity of some form of Penrose's cosmic censorship conjecture, and proving the stability and, thus, the physical relevance, of the known general relativity black hole solutions. Although the first such solution was found by Karl Schwarzschild a few months after Einstein's field equations were published, its stability under perturbations has not yet been fully established. Even the proof of the linear stability of the Schwarzschild black hole remains incomplete, as only its modal linear stability, defined as the boundedness of the potentials that generate isolated harmonic modes, has been proved. In this Letter, we show that the geometric information of the most general linear perturbation is encoded in two spacetime scalar fields $\Phi_{ \pm}$made from perturbed curvature invariants. These fields measure the distortion of the geometry and are shown to be pointwise bounded. Besides giving a definite answer to the problem of the linear stability of the Schwarzschild black hole, the techniques we introduce are likely to apply to the rotating Kerr black hole [1].

The linearized Einstein's equation (LEE) describes the propagation of a perturbation $\delta g_{\alpha \beta}$ of the metric as a wave on the background spacetime $\left(M, g_{\alpha \beta}\right)$

$$
\nabla^{\gamma} \nabla_{\gamma} \delta g_{\alpha \beta}+\nabla_{\alpha} \nabla_{\beta}\left(g^{\gamma \delta} \delta g_{\gamma \delta}\right)-2 \nabla^{\gamma} \nabla_{(\alpha} \delta g_{\beta) \gamma}=0
$$

Trivial solutions of this equations are obtained by relabeling the points of $M$ by means of an infinitesimal diffeomorphism $V^{\alpha}$, under which

$$
g_{\alpha \beta} \rightarrow g_{\alpha \beta}+\mathfrak{E}_{V} g_{\alpha \beta}=g_{\alpha \beta}+\nabla_{\alpha} V_{\beta}+\nabla_{\beta} V_{\alpha} .
$$

Two solutions of Eq. (1) that differ by $£_{V} g_{\alpha \beta}=\nabla_{\alpha} V_{\beta}+$ $\nabla_{\beta} V_{\alpha}$ are, therefore, physically equivalent; this is the gauge invariance of linearized gravity. The problem of linear stability of a stationary vacuum metric $g_{\alpha \beta}$ is that of finding out whether the effects on the geometry of a solution of Eq. (1) are bounded by the initial data of the wave or not.

The study of the linear stability of a Schwarzschild black hole $\mathcal{M}$ has a long history that dates back to the 1957 pioneering work of Regge and Wheeler [2], where the spherical symmetry of the background was used to split the metric perturbation into what were termed even $(+)$ and odd (-) modes of harmonic numbers $(l, m)$. Since these modes do not mix at the linear level, pure modes are analyzed. A master variable $\phi_{(\ell, m)}^{ \pm}$defined on the $(t, r)$ space [the orbit space $\mathcal{M} / S O(3)$ ] is extracted for single modes, and the LEE is reduced to a two-dimensional scalar wave equation on the orbit space

$$
\frac{\partial^{2} \phi_{(\ell, m)}^{ \pm}}{\partial t^{2}}+\mathcal{H}_{\ell}^{ \pm} \phi_{(\ell, m)}^{ \pm}=0, \quad \mathcal{H}_{\ell}^{ \pm} \equiv-\frac{\partial^{2}}{\partial x^{2}}+f U_{\ell}^{ \pm}
$$

where $f=1-2 M / r$ and $x$ is a "tortoise" radial coordinate, defined by $d x / d r=1 / f$. For the odd modes Eq. (3), known as the Regge-Wheeler equation, was worked out in Ref. [2], and the potential is

$$
U_{\ell}^{-}=\left(\frac{\ell(\ell+1)}{r^{2}}-\frac{6 M}{r^{3}}\right) .
$$

The more intricate system of even perturbations was simplified to the form of Eq. (3) by Zerilli in 1970 [3]; the potential for these modes is

$$
U_{\ell}^{+}=\frac{\mu^{2} r^{2}\left[\left(\mu^{2}+2\right) r+6 M\right]+36 M^{2}\left(\mu^{2} r+2 M\right)}{r^{3}\left(\mu^{2} r+6 M\right)^{2}},
$$

where $\mu^{2}=(\ell+2)(\ell-1)$. Note that $V_{\ell}^{ \pm} \equiv f U_{\ell}^{ \pm}$are positive and go to zero at both the $x \rightarrow-\infty$ (black hole horizon) and $x \rightarrow \infty$ (spacelike infinity) limits; thus, $\mathcal{H}_{\ell}^{ \pm}$in 
Eq. (3) is formally equivalent to a positive definite quantum Hamiltonian acting on the space of square integrable functions of $x$. The gauge invariance of $\phi_{(\ell, m)}^{ \pm}$was proved by Moncrief in Ref. [4]; Sengupta and Gerlach [5] showed how to recast the Regge-Wheeler equations and their relation to odd metric perturbations as covariant equations in the twodimensional orbit space (for a quick review, see Section II of Ref. [6], where the even sector is worked out). If we write the Schwarzschild metric as [5]

$$
g_{\alpha \beta} d z^{\alpha} d z^{\beta}=g_{a b} d x^{a} d x^{b}+r^{2}(x) \hat{g}_{i j} d y^{i} d y^{j},
$$

where $\hat{g}_{i j} d y^{i} d y^{j}=d \theta^{2}+\sin ^{2} \theta d \phi^{2}$ is the metric on the unit sphere and $g_{a b} d x^{a} d x^{b}$ is the Lorentzian metric on the orbit space [given by $-(1-2 M / r) d t^{2}+d r^{2} /(1-2 M / r)$ in Schwarzschild coordinates] and let $g^{a b}, D_{b}, \epsilon_{a b}$ be the inverse metric, covariant derivative, and volume form of the orbit space and $\hat{g}^{i j}, \hat{D}_{k}, \hat{\epsilon}_{i j}$ those of the unit sphere, then the Regge-Wheeler and Zerilli equations (3) read

$$
g^{a b} D_{a} D_{b} \phi_{(\ell, m)}^{ \pm}-U_{\ell}^{ \pm} \phi_{(\ell, m)}^{ \pm}=0 .
$$

To reconstruct the metric perturbation, a real orthonormal basis of spherical harmonics $S_{(\ell, m)}$ is used,

$$
\hat{D}^{i} \hat{D}_{i} S_{(\ell, m)}=-\ell(\ell+1) S_{(\ell, m)} .
$$

Odd $\ell>1$ modes in the Regge-Wheeler gauge are

$$
\begin{gathered}
\delta g_{a i}^{(\ell, m,-)}=\left[\epsilon_{a c} g^{c b} D_{b}\left(r \phi_{(\ell, m)}^{-}\right)\right]\left[\hat{\epsilon}_{k i} \hat{g}^{k j} \hat{D}_{j} S_{(\ell, m)}\right], \\
\delta g_{a b}^{(\ell, m,-)}=0, \quad \delta g_{i j}^{(\ell, m,-)}=0
\end{gathered}
$$

where $\phi_{(\ell, m)}^{-}$is an arbitrary real solution of Eq. (7). For $\ell=1$,

$$
J_{m}{ }^{l}=\hat{g}^{l i}\left[\hat{\epsilon}_{k i} \hat{g}^{k j} \hat{D}_{j} S_{(\ell, m)}\right]
$$

is a basis of Killing vectors on the sphere, tangent to rotations around three orthogonal axes (e.g., $J_{3}=\partial / \partial \phi$ ), and a gauge can be found such that the only nonzero components of an arbitrary linear combination of the $\delta g_{a i}^{(1, m,-)}$ in standard coordinates are [see equation (11) in Ref. [6]]

$$
\delta g_{t i}^{(1,-)}=-r^{-1} \sum_{m=1}^{3} \delta a^{m} J_{m i} .
$$

This perturbation corresponds to turning on an infinitesimal angular momentum in the direction $\delta \vec{a}=\left(\delta a^{1}, \delta a^{2}, \delta a^{3}\right)$. The odd $\ell=0$ mode is void, whereas the even $\ell=0$ mode amounts to a change of the black hole mass

$$
\delta g_{t t}^{(0,+)}=\frac{2 \delta M}{r}, \quad \delta g_{r r}^{(0,+)}=\frac{2 \delta M}{r(1-2 M / r)^{2}} .
$$

Even $\ell=1$ modes are pure gauge, whereas higher even modes are involved expressions on $\left(\phi_{(\ell, m)}^{+}, S_{(\ell, m)}\right)$ that we need not spell out here (see, e.g., Refs. [5,6]).

The Zerilli and Regge-Wheeler equations allow us to analyze isolated modes and to establish a basic notion of linear stability, based on the fact that they admit separable solutions of the form $\phi=e^{\alpha t} \chi(x)$ (we suppress indices for simplicity). From Eq. (3), it can easily be shown that $\alpha$ must be purely imaginary, $\alpha=i \omega$, since $\alpha^{2} \chi=-\mathcal{H} \chi$ and $\mathcal{H}$ is positive definite. Since the perturbation of any geometric field is obtained by applying a linear differential operator to $\delta g_{\alpha \beta}$, it will also be oscillatory and, thus, bounded in time. Exponential growth for nonseparable solutions of Eq. (3) can be ruled out using the positive definite conserved energy of Eq. (3). An integral bound of the form $\int\left|\phi_{(\ell, m)}(t, x)\right|^{2} d x \leq C_{(\ell, m)}$ was obtained in Ref. [7], where the possibility of unbounded growth in narrowing intervals was also ruled out by proving that a pointwise bound can be placed on the $\phi_{(\ell, m)}^{ \pm}$

$$
\left|\phi_{(\ell, m)}^{ \pm}\right| \leq K_{(\ell, m)}^{ \pm}, \quad r>2 M,
$$

with $K_{(\ell, m)}^{ \pm}$a constant obtained from the $(\ell, m, \pm)$ piece of the initial data.

Nonmodal stability.-The most general linear perturbation of the Schwarzschild black hole is of the form

$$
\delta g_{\alpha \beta}^{(1,-)}(\delta \vec{a})+\delta g_{\alpha \beta}^{(0,+)}(\delta M)+\sum_{\substack{m, P= \pm \ell \geq 2}} \delta g_{\alpha \beta}^{(\ell, m, P)}[\phi, S] .
$$

The first two terms in Eq. (15), given in Eqs. (12) and (13), are deviations within the Kerr family and are time independent: no dynamical process can lead to a change in mass or angular momentum in the linear regime; these processes show up at second order [8]. The series in Eq. (15), whose completeness follows from the theorems in Section 2.3 of Ref. [9], does not contribute to any of these charges and encodes the dynamics of the perturbation. The $\phi_{(\ell, m)}^{ \pm}$are an infinite set of potentials whose derivatives enter individual terms in this series, and two extra derivatives must be taken to calculate the perturbed Riemann tensor and analyze the effects of the perturbation.

In order to evaluate whether a perturbation "grows big" or not, we first need to parametrize the space $\mathcal{L}$ of solutions of the LEE (1) with geometrically meaningful quantities. For this purpose, it is important to understand how the different modes in Eq. (15) behave under the action of the symmetry group of the Schwarzschild metric. The isometries of the background commute with the LEE, Eq. (1). When applied to a linearized solution $g_{\alpha \beta}+\delta g_{\alpha \beta}$, they keep $g_{\alpha \beta}$ fixed while acting on $\delta g_{\alpha \beta}$ as linear operators in $\mathcal{L}$. The 
isometry group of the Schwarzschild metric is $\mathbb{R}_{t} \times T \times S O(3) \times P$, where $\mathbb{R}_{t}$ is the subgroup of time translations, $S O(3)$ are the proper rotations, and $T$ and $P$ are the $\mathbb{Z}_{2}$ subgroups of time inversion $T:(t, r, \theta, \phi) \rightarrow$ $(-t, r, \theta, \phi)$ and parity transformation $P:(\theta, \phi) \rightarrow(\pi-$ $\theta, \phi+\pi)$. The $(\ell, m)$ labels are attached to modes constructed from the $S_{(\ell, m)}$ spherical harmonics and their first and second derivatives. These derivatives are the components of eigentensors of the Laplacian on the sphere [9].

The "square angular momentum" operator

$$
\mathbf{J}^{2}=\sum_{m=1}^{3}\left(£_{J_{m}}\right)^{2}
$$

acting on Eq. (9) gives

$$
\mathbf{J}^{2} \delta g_{\alpha \beta}^{(\ell, m, \pm)}=-\ell(\ell+1) \delta g_{\alpha \beta}^{(\ell, m, \pm)},
$$

and if we used the standard spherical harmonics $Y_{(\ell, m)}$ instead of a real basis, $\delta g_{\alpha \beta}^{(\ell, m, \pm)}$ would also be a (complex) eigentensor of $£_{J_{3}}$, with eigenvalue $\mathrm{im}$. The meaning of "even" and "odd" modes (introduced with quotes in the original work [2]) is more obscure; it tells us whether the perturbation behaves as a scalar field or not

$$
P_{*} \delta g_{\alpha \beta}^{(\ell, m, \pm)}= \pm(-1)^{\ell} \delta g_{\alpha \beta}^{(\ell, m, \pm)} .
$$

A more significant interpretation can be given, as we now proceed to explain. For vacuum spacetimes, every algebraic invariant of the Riemann tensor can be written as a polynomial in four basic invariants

$$
\begin{aligned}
& Q=\frac{1}{48}\left(C_{\alpha \beta \gamma \delta}+i C_{\alpha \beta \gamma \delta}^{*}\right) C^{\alpha \beta \gamma \delta}=Q_{+}+i Q_{-}, \\
& C=\frac{1}{96}\left(C_{\alpha \beta}{ }^{\gamma \delta}+i C_{\alpha \beta}^{* \gamma \delta}\right) C_{\gamma \delta}{ }^{\epsilon \mu} C_{\epsilon \mu}{ }^{\alpha \beta}=C_{+}+i C_{-},
\end{aligned}
$$

where $C_{\alpha \beta \gamma \delta}$ is the Weyl tensor and $C_{\alpha \beta \gamma \delta}^{*}:=\frac{1}{2} \epsilon_{\alpha \beta}{ }^{\epsilon \mu} C_{\epsilon \mu \gamma \delta}$ is its dual. Note that $\epsilon_{\alpha \beta \gamma \delta}$, the volume form of spacetime, is odd under parity, $P_{*} \epsilon_{\alpha \beta \gamma \delta}=-\epsilon_{\alpha \beta \gamma \delta}$, and that $Q_{-}$and $C_{-}$are "pseudoscalar"; i.e., their construction requires the volume form besides the metric, they are orientation dependent and pick up an extra minus sign under $P$, their $(\ell, m)$ piece transforming as $(-1)^{\ell+1}$. For the Schwarzschild spacetime,

$$
Q=\frac{M^{2}}{r^{6}}, \quad C=\frac{M^{3}}{r^{9}},
$$

the vanishing of the pseudoscalars being forced by the facts that they must be odd under $P$ but cannot depend on the angular variables (since they must vanish under $£_{J_{k}}$ ). In addition to $Q$ and $C$, differential invariants of the Weyl tensor are required to fully characterize a vacuum metric, the simplest one being

$$
X=\frac{1}{720}\left(\nabla_{\varepsilon} C_{\alpha \beta \gamma \delta}\right)\left(\nabla^{\varepsilon} C^{\alpha \beta \gamma \delta}\right)=\frac{M^{2}}{r^{9}}(r-2 M) .
$$

Under a perturbation, the first-order variation $\delta I_{ \pm}$of a (pseudo) scalar invariant $I_{ \pm}$of the Weyl tensor (such as $Q$, $C, X$, and $Y$ above) is a linear functional of $\delta g_{\alpha \beta}$ that commutes with all symmetries, and then

$$
\begin{aligned}
(-1)^{\ell} \delta I_{+}\left[\delta g_{\alpha \beta}^{(\ell, m,-)}\right] & =P \delta I_{+}\left[\delta g_{\alpha \beta}^{(\ell, m,-)}\right] \\
& =\delta I_{+}\left[P_{*} \delta g_{\alpha \beta}^{(\ell, m,-)}\right] \\
& =-(-1)^{\ell} \delta I_{+}\left[\delta g_{\alpha \beta}^{(\ell, m,-)}\right],
\end{aligned}
$$

which implies that $\delta I_{+}\left[\delta g_{\alpha \beta}^{(\ell, m,-)}\right]=0 . \quad$ Similarly, $\delta I_{-}\left[\delta g_{\alpha \beta}^{(\ell, m,+)}\right]$ must be zero. Thus, (odd) even perturbations can be better characterized as those exciting perturbations of curvature (pseudo) scalars.

If we calculate $\delta Q_{-}\left[\delta g_{\alpha \beta}^{(\ell, m,-)}\right]$ from Eq. (15), we get a rather complicated expression which simplifies if we make use of the LEE together with their derivatives, leaving a strikingly simple expression

$$
\delta Q_{-}=\frac{-6 M^{2}}{r^{7}} S_{(1, m)} \delta a^{m}+\frac{3 M}{r^{6}} \sum_{\ell>1, m} \frac{(\ell+2) !}{(\ell-2) !} \phi_{(\ell, m)}^{-} S_{(\ell, m)} .
$$

The above equation shows that the local curvature pseudoscalar $\delta Q_{-}$encodes all the information carried by the most general odd perturbation, as the nonlocal quantities $\delta \vec{a}$ and $\phi_{(\ell, m)}^{-}$can be recovered by integrating $\delta Q_{-}$against $S_{(\ell, m)}$ on the sphere. Moreover, Eq. (22) together with Eqs. (7) and (8) implies that

$$
\Phi_{-} \equiv \frac{r^{5}}{3 M} \delta Q_{-}
$$

satisfies a simple four-dimensional wave equation on the Schwarzschild spacetime

$$
\left[\nabla_{\alpha} \nabla^{\alpha}+\frac{8 M}{r^{3}}\right] \Phi=0
$$

$\left(\Phi=\Phi_{-}\right)$. This equation is also satisfied by the potential $\Phi_{-}^{o}=\sum_{\ell \geq 2, m}\left(\phi_{(\ell, m)}^{-} / r\right) S_{(\ell, m)}$, in terms of which the dynamical terms of the metric perturbation (9) can be compactly written in a covariant way as

$$
\sum_{(\ell>1, m)} \delta g_{\alpha \beta}^{(\ell, m,-)}=\frac{r^{2}}{3 M}{ }^{*} C_{\alpha}{ }^{\gamma \delta}{ }_{\beta} \nabla_{\gamma} \nabla_{\delta}\left(r^{3} \Phi_{-}^{o}\right) .
$$

Theorem: Nonmodal linear stability of the Schwarzschild black hole (odd sector).--The space $\mathcal{L}_{-}$of solutions of the 
LEE around a Schwarzschild black hole mod gauge transformations is parametrized by the gauge invariant pseudoscalar field $\delta Q_{-}$. For any perturbation with compact support on Cauchy surfaces of the Kruskal extension,

$$
\left|\delta Q_{-}\right|<K_{-} / r^{6},
$$

on the exterior wedge $r \geq 2 M$, with $K_{-}$a constant that depends on the perturbation data on a $t$ slice.

Proof: The only thing that remains is to prove the bound (26); $\Phi_{-}$satisfies Eq. (24) which, following Ref. [10] where the Klein Gordon equation on the Schwarzschild background is studied, can be written as $0=\left(\partial_{t}^{2}-\partial_{x}^{2}+\right.$ $\left.V_{1}-\hat{D}^{k} \hat{D}_{k} V_{2}\right)(r \Phi)$. We find from Eq. (4) that $V_{1}=-(1-$ $2 M / r) 6 M / r^{3}$ and $V_{2}=(1-2 M / r) / r^{2}$ are both bounded for $r \geq 2 M$; therefore, the proof in Appendix A of Ref. [10] applies to Eq. (24) as well as the symmetry argument in the main text and implies that $\left|\Phi_{-}\right|<K_{-}^{\prime} / r$, from where Eq. (26) follows.

Even perturbations are more difficult to deal with for two reasons. (i) The dependence of Eq. (5) on $\ell$ indicates that the set of Zerilli functions $\phi_{(\ell, m)}^{+}$is not directly related to the harmonic components of a four-dimensional scalar field and (ii) although the scalar invariant $Q_{+}$is excited by the even modes, the excitations $\delta Q_{+}$are not gauge invariant because $Q_{+}$does not vanish in the background and, under the gauge transformation (2),

$$
\delta Q_{+} \rightarrow \delta Q_{+}+£_{V} Q_{+}=\delta Q_{+}+V^{r} \partial_{r} Q_{+}
$$

and similarly for $C_{+}$. Problem (ii) is absent in the odd sector because $Q_{-}=0=C_{-}$. To tackle it, we could substitute $\delta Q_{+}$with any gauge invariant combination of perturbed scalars. However, when computing $\delta Q_{+}$and $\delta C_{+}$in the Regge-Wheeler (RW) gauge we find that $\delta Q_{+} / \delta C_{+}=$ $\partial_{r} C_{+} / \partial_{r} Q_{+}$, and this fact, together with Eq. (27), implies that all such gauge invariants will vanish under a genuine perturbation and so are useless. Thus, we need to incorporate differential invariants, such as $X$ in Eq. (21), which do not satisfy simple equations. The simplest gauge invariant combination of the enlarged set of perturbed scalars is

$$
\Phi_{+}=(9 M-4 r) \delta Q_{+}+3 r^{3} \delta X .
$$

We will use it to measure the effect of even perturbations on the geometry. To deal with reason (i), we use the factorization property [11]

$$
\begin{aligned}
& \mathcal{H}_{\ell}^{ \pm}=\mathcal{A}_{\ell}^{ \pm} \mathcal{A}_{\ell}^{\mp}-E_{\ell}^{2}, \quad \mathcal{A}_{\ell}^{ \pm}= \pm \frac{\partial}{\partial x}+W_{\ell}, \\
& W_{\ell}=E_{\ell}+\frac{6 M(r-2 M)}{r^{2}((\ell+2)(\ell-1) r+6 M)}, \\
& E_{\ell}=\frac{1}{12 M} \frac{(\ell+2) !}{(\ell-2) !} .
\end{aligned}
$$

Thus, $\mathcal{A}_{+} \phi_{(\ell, m)}^{-}$solves the even Eq. (3) if $\phi_{(\ell, m)}^{-}$solves the odd one. This suggest that we write even metric perturbations using odd potentials through $\phi_{(\ell, m)}^{+}=\mathcal{A}_{+} \phi_{(\ell, m)}^{-}$. A lengthy calculation using the LEE then reduces $\Phi_{+}$in Eq. (28) to

$$
\begin{gathered}
-\frac{2 M \delta M}{r^{5}}+\sum_{3 \leq j \leq 7} \frac{M^{j-3}}{r^{j}}\left[M \partial_{r} \Phi_{(1, j)}+M^{2} \partial_{t}^{2} \Phi_{(2, j)}+\Phi_{(3, j)}\right] \\
\Phi_{(k, j)}=\sum_{(\ell \geq 2, m)} P_{(k, j)} \frac{\phi_{(\ell, m)}^{-}}{r} S_{(\ell, m)}, \quad 1 \leq k \leq 3
\end{gathered}
$$

where $P_{(k, j)}$ are polynomials in $\ell$.

Theorem: Nonmodal linear stability of the Schwarzschild black hole (even sector). - The space $\mathcal{L}_{+}$ of solutions of the linearized Einstein equations around a Schwarzschild black hole mod gauge transformations is parametrized by the gauge invariant scalar field $\Phi_{+}$in Eq. (28). For any perturbation with compact support on Cauchy surfaces of the Kruskal extension,

$$
\left|\Phi_{+}\right|<K_{+} / r^{3}
$$

on the exterior wedge $r \geq 2 M$, with $K_{+}$a constant that depends on the perturbation data on a $t$ slice.

Proof: A generic even perturbation is parametrized by the Zerilli potentials entering $\delta g_{\alpha \beta}^{(\ell \geq 2, m,+)}$ and $\delta M$ [see Eq. (15)]. In terms of these, using the LEE one finds

$$
\begin{aligned}
\Phi_{+} & =-\frac{2 M \delta M}{r^{5}}+\frac{M}{2 r^{4}} \sum_{\ell \geq 2} \frac{(\ell+2) !}{(\ell-2) !}\left[\partial_{x}+Z_{\ell}(x)\right] \phi_{(\ell, m)}^{+} S_{(\ell, m)}, \\
Z_{\ell} & =\frac{\mu r(r-3 M)-6 M^{2}}{r^{2}(\mu r+6 M)}, \quad \mu=(\ell-1)(\ell+2)
\end{aligned}
$$

By expanding $\Phi_{+}$in spherical harmonics we get $\delta M$ and $\left[\partial_{x}+Z_{\ell}(x)\right] \phi_{(\ell, m)}^{+}$, from where $\phi_{(\ell, m)}^{+}$can be solved. The parametrization of $\mathcal{L}_{+}$then follows. The alternative form (31), obtained by replacing $\phi_{(\ell, m)}^{+}=\mathcal{A}_{+} \phi_{(\ell, m)}^{-}$in Eq. (33), is used to prove Eq. (32). Every $\Phi_{(k, j)}$ field in Eq. (31) and then every $\partial_{t}^{2} \Phi_{(k, j)}$ field satisfies the wave equation Eq. (24); its absolute value is then bounded by a constant times $r^{-1}$, and its $r$ derivative is bounded by a constant on the $r \geq 2 M$ region, as proved by a direct transcription of the results in Section 3.6 of Ref. [12]. Then, Eq. (32) follows.

In conclusion, we have shown the following. (i) The information on arbitrary metric perturbations is contained in $\Phi_{ \pm}$given in Eqs. (23) and (28). These are gauge invariant curvature scalars that can be measured locally, unlike RW and Zerilli potentials, which require integrations on the sphere. (ii) For generic perturbations, the initial data places a pointwise bound for $\Phi_{ \pm}$in the outer region. 
I would like to thank Sergio Dain, Reinaldo Gleiser, Jacek Jezierski, and Robert Wald for pointing out errors in the first version of this manuscript. This work was partially funded from Grants No. PICT-2010-1387, No. PIP 11220080102479, and No. Secyt-UNC 05/B498. The GRTENSOR package (grtensor.org) was used to calculate perturbed curvature invariants.

*gdotti@famaf.unc.edu.ar

[1] B. Araneda and G. Dotti (to be published).

[2] T. Regge and J. A. Wheeler, Phys. Rev. 108, 1063 (1957).

[3] F. J. Zerilli, Phys. Rev. Lett. 24, 737 (1970).

[4] V. Moncrief, Ann. Phys. (N.Y.) 88, 323 (1974).
[5] U. H. Gerlach and U. K. Sengupta, Phys. Rev. D 19, 2268 (1979).

[6] O. Sarbach and M. Tiglio, Phys. Rev. D 64, 084016 (2001).

[7] R. M. Wald, J. Math. Phys. (N.Y.) 20, 1056 (1979); 21, 218(E) (1980).

[8] R. J. Gleiser, Classical Quantum Gravity 14, 1911 (1997); G. Khanna, R. Gleiser, R. Price, and J. Pullin, New J. Phys. 2, 3 (2000).

[9] A. Ishibashi and H. Kodama, Prog. Theor. Phys. Suppl. 189, 165 (2011).

[10] B. S. Kay and R. M. Wald, Classical Quantum Gravity 4, 893 (1987).

[11] S. Chandrasekhar, The Mathematical Theory of Black Holes (Clarendon, Oxford, England, 1983), p. 160.

[12] S. Dain and G. Dotti, Classical Quantum Gravity 30, 055011 (2013). 